\title{
Market Making with Alpha Signals
}

\author{
Álvaro Cartea ${ }^{\mathrm{a}}$, Yixuan Wang ${ }^{\mathrm{b}}$ \\ ${ }^{a}$ Mathematical Institute, University of Oxford, Oxford, UK \\ Oxford-Man Institute of Quantitative Finance, Oxford, UK \\ ${ }^{b}$ Mathematical Institute, University of Oxford, Oxford, UK
}

\begin{abstract}
We show how a market maker employs information about the momentum in the price of the asset (i.e., alpha signal) to make decisions in their liquidity provision strategy in an order driven electronic market. The momentum in the midprice of the asset depends on the execution of liquidity taking orders and the arrival of news. Buy market orders (MOs) exert a short-lived upward pressure on the midprice, whereas sell MOs exert a short-lived downward pressure on the midprice. We employ Nasdaq high-frequency data to estimate model parameters and to illustrate the performance of the market making strategy. The market maker employs the alpha signal to minimise adverse selection costs, execute directional trades in anticipation of price changes, and to manage inventory risk. As the market maker increases their tolerance to inventory risk, the expected profits that stem from the alpha signal increase because the strategy employs more speculative MOs and performs more roundtrip trades with limit orders.
\end{abstract}

Keywords: Market making; alpha signal; high-frequency trading; momentum trading; order flow; adverse selection; latent alpha

\section{Introduction}

In this paper we show how a market maker employs information about the momentum in the price of the asset to provide liquidity in an order driven electronic market. The midprice of the asset is modelled by a pure jump process and we refer to the arrival rate of the midprice innovations as the alpha signal. Liquidity taking orders, news, and other information in the marketplace affect the alpha signal. When a buy market order (MO) arrives in the exchange, the alpha signal jumps up and when a sell MO arrives in the exchange, the alpha signal jumps

Email addresses: Alvaro.Cartea@maths.ox.ac.uk (Álvaro Cartea), yixuan.wang@maths.ox.ac.uk (Yixuan Wang) 
down. The alpha signal also undergoes diffusive shocks that represent other events that affect the limit order book (LOB), in particular the best bid and best ask prices of the asset. When the value of the alpha signal is positive (negative), it is more likely to observe positive (negative) price innovations - the sign of the alpha signal determines the momentum in the midprice of the asset.

The market maker maximises terminal expected wealth, while penalising inventory holdings throughout the life of the trading strategy. We formulate the problem as a stochastic and impulse control problem and derive the corresponding Hamilton-Jacobi-Bellman Quasi-Variational Inequality (HJBQVI), which we solve numerically.

We use Nasdaq high-frequency data to estimate the parameters of the model and run simulations to show the performance of the strategy. When inventory is close to zero and the alpha signal is near zero, the market maker posts both sell and buy limit orders (LOs) - this is optimal because the arrival rates of upward and downward jumps in the midprice is approximately the same, so there is zero momentum in the midprice. As the value of the alpha signal increases, the market maker posts buy LOs and does not post sell LOs to minimise adverse selection costs, i.e., to avoid being picked off by high-frequency traders. When the value of the alpha signal increases beyond a critical level, the market maker executes buy MOs in anticipation of an imminent increase in the price of the asset. This expected increase in price is large enough to cover the costs that stem from liquidity taking fees (i.e., exchange fees) and from crossing the spread - the behaviour of the strategy is similar when the value of the alpha signal is negative and inventory is close to zero.

As the market maker increases their tolerance to inventory risk, the expected profits that stem from employing the alpha signal increase because the strategy employs more speculative MOs in the direction of the expected change in prices and performs more roundtrip trades with LOs. On the other hand, these speculative directional trades and additional LOs increase the volatility of the inventory, and consequently, the volatility of the profits of the strategy also increase. The optimal strategy trades off expected profits and the volatility of the inventory holdings. We show that there is a range of tolerance to inventory risk where the expected profits of the strategy increase and the volatility of the profits of the strategy hardly changes as the tolerance to inventory risk increases. Finally, if the market maker's tolerance to inventory risk is very low, the strategy benefits very little from the alpha signal and, all else being equal, the expected profits of the strategy are lowest.

There is abundant literature on market making. Ho et al. (1981) consider the problem of optimal quoting, where the market maker decides the optimal depth of the bid and ask prices, relative to the price of the asset. The market maker assumes there is a linear-decay relation between the depth and the intensity of the market orders. Avellaneda and Stoikov (2008) consider a similar problem where they assume an exponential decay relation between the depth of LOs and the 
arrival intensity of MOs. In a similar vein, Guéant et al. (2013) solve the problem of a market maker who imposes bounds on the inventory holdings. Cartea et al. (2014) consider a model that accounts for the dependence between MOs, LOB dynamics, and midprice innovations; see also Cartea et al. (2018). The work of Cartea and Jaimungal (2015) introduces several risk metrics that the market maker employs to trade off inventory risk against expected profits. In Cartea et al. (2017) the authors allow for model ambiguity to make the market making model robust to misspecification in the: arrival rate of MOs, fill probability of LOs, and midprice dynamics.

The remainder of the paper is organized as follows. Section 2 introduces the model setup, shows how to estimate the parameters of the model, and illustrates some features of the optimal market making strategy. Section 3 presents simulations of the optimal strategy and discusses the distributions of the profit and loss (P\&L) and of the number of filled LOs and executed MOs. Section 5 introduces the numerical scheme and shows convergence results. Finally, Section 6 concludes.

\section{Model}

We fix a complete filtered probability space $\left(\Omega, \mathcal{F},\left\{\mathcal{F}_{t}\right\}_{t \in[0, T]}, \mathbb{P}\right)$, where $\left\{\mathcal{F}_{t}\right\}_{t \in[0, T]}$ is the natural filtration generated by the collection of observable stochastic processes that we define below. The market maker trades over the time window $[0, T]$, where the terminal time $T$ is a positive constant.

Let $S=\left(S_{t}\right)_{t \geq 0}$ denote the midprice of the asset and assume it follows the pure jump process

$$
\mathrm{d} S_{t}=\sigma\left(\mathrm{d} J_{t}^{+}-\mathrm{d} J_{t}^{-}\right)
$$

where $\sigma>0$ is the size of the tick in the LOB. Here, $J_{t}^{+}$and $J_{t}^{-}$are doubly stochastic Poisson processes with intensities $\mu_{t}^{+}$and $\mu_{t}^{-}$, respectively, and given by

$$
\mu_{t}^{+}=\left(\alpha_{t}\right)_{+}+\theta \quad \text { and } \quad \mu_{t}^{-}=\left(\alpha_{t}\right)_{-}+\theta
$$

where $\theta>0$ is the baseline arrival intensity of the midprice innovations.

The process $\alpha=\left(\alpha_{t}\right)_{t \geq 0}$ is the alpha signal and $(\cdot)_{+}$and $(\cdot)_{-}$are operators that take the absolute value of the positive and negative parts of the argument, so that $\alpha_{t}=\left(\alpha_{t}\right)_{+}-\left(\alpha_{t}\right)_{-}$.

Other market participants send buy and sell MOs to the electronic exchange to trade the asset. Buy MOs arrive with intensity $\lambda^{+}$and sell MOs arrive with intensity $\lambda^{-}$. Let $M^{0+}=\left(M_{t}^{0+}\right)_{t \geq 0}$ and $M^{0-}=\left(M_{t}^{0-}\right)_{t \geq 0}$ denote the counting processes of buy and sell MOs executed by other market participants - below we provide details of the effect that MOs have on the alpha signal. 
The market maker decides when to post buy and sell LOs. The control process $l_{t}^{+} \in\{0,1\}$ represents the activity of sell LOs and $l_{t}^{-} \in\{0,1\}$ represents the activity of buy LOs. When $l_{t}^{+}=1$, the market maker posts a sell LO and when $l_{t}^{+}=0$, the strategy does not post a sell LO; the control for a buy LO is similar. Sell and buy LOs are of volume one and are posted at the best ask and best bid prices $S_{t}+\Delta$ and $S_{t}-\Delta$, respectively, where $\Delta \geq 0$ denotes the half-spread. Conditioned on the arrival of a buy (sell) MO from other participants, the sell (buy) LO posted by the market maker is filled with probability $p^{+}\left(p^{-}\right) \in(0,1]$. The processes $N^{+, l}=\left(N_{t}^{+, l}\right)_{t \geq 0}$ and $N^{-, l}=\left(N_{t}^{-, l}\right)_{t \geq 0}$ count the number of filled sell and buy LOs by the market maker.

The vectors $\boldsymbol{\tau}^{+}=\left(\tau_{1}^{+}, \tau_{2}^{+}, \tau_{3}^{+} \ldots\right)$ and $\boldsymbol{\tau}^{-}=\left(\tau_{1}^{-}, \tau_{2}^{-}, \tau_{3}^{-} \ldots\right)$ represent the times when the market maker sends MOs, where the superscript + denotes buy MOs and the superscript denotes sell MOs. The market maker sends MOs of volume one. The counting processes for the market maker's buy and sell MOs are denoted by $M^{+}=\left(M_{t}^{+}\right)_{t \geq 0}$ and $M^{-}=\left(M_{t}^{-}\right)_{t \geq 0}$ and are given by

$$
M_{t}^{+}=\sum_{k=1}^{\infty} \mathbb{1}_{\left\{\tau_{k}^{+} \leq t\right\}} \quad \text { and } \quad M_{t}^{-}=\sum_{k=1}^{\infty} \mathbb{1}_{\left\{\tau_{k}^{-} \leq t\right\}} .
$$

Buy and sell MOs execute at prices $S_{t}+\Upsilon_{M O}$ and $S_{t}-\Upsilon_{M O}$, respectively, where the liquidity taking costs are $\Upsilon_{M O}=\Delta+\varepsilon_{M O}$ and $\varepsilon_{M O}>0$ is the exchange fee. We denote the control of the market maker by $\nu=\left(l^{ \pm}, \boldsymbol{\tau}^{ \pm}\right)$.

The assumptions about the volume of the market maker's LOs and MOs can be easily relaxed. For example, the volume of the MOs and LOs could be greater than one, and in particular, we can extend the model to one in which the market maker controls the volume of each LO and each $\mathrm{MO}$ they sends to the exchange. For the scope of this paper, we assume that conditioned on the arrival of a MO, the LOs posted by the market maker are filled with probabilities $p^{+}=p^{-}=1$.

Finally, the alpha signal follows the dynamics

$$
\mathrm{d} \alpha_{t}^{\nu}=-\kappa \alpha_{t}^{\nu} \mathrm{d} t+\xi \mathrm{d} W_{t}+\eta^{+}\left(\mathrm{d} M_{t}^{0+}+\mathrm{d} M_{t}^{+}\right)-\eta^{-}\left(\mathrm{d} M_{t}^{0-}+\mathrm{d} M_{t}^{-}\right), \quad \alpha_{0}=0,
$$

where $W=\left(W_{t}\right)_{t \geq 0}$ is a standard Brownian motion, and $\kappa, \xi, \eta^{+}, \eta^{-}$are positive constants. Observe that the arrival of MOs in the exchange cause the alpha signal to jump up by $\eta^{+}$if the MO is a buy order or jump down by $\eta^{-}$if the MO is a sell order. The Brownian component of the alpha signal represents the effect of the arrival of news in the market and other information that liquidity providers employ in their strategies.

The market maker's controlled inventory is denoted by $Q^{\nu}=\left(Q_{t}^{\nu}\right)_{t \geq 0}$ with dynamics

$$
Q_{t}^{\nu}=N_{t}^{-, l}-N_{t}^{+, l}+M_{t}^{+}-M_{t}^{-},
$$


and the inventory obeys the bounds $-\bar{Q} \leq Q_{t}^{\nu} \leq \bar{Q}$ for some integer $\bar{Q}>0$. The controlled cash process is denoted by $X^{\nu}=\left(X_{t}^{\nu}\right)_{t \geq 0}$ and satisfies

$$
\mathrm{d} X_{t}^{\nu}=\left(S_{t}^{\nu}+\Upsilon_{L O}\right) \mathrm{d} N_{t}^{+, l}-\left(S_{t}^{\nu}-\Upsilon_{L O}\right) \mathrm{d} N_{t}^{-, l}-\left(S_{t}^{\nu}+\Upsilon_{M O}\right) \mathrm{d} M_{t}^{+}+\left(S_{t}^{\nu}-\Upsilon_{M O}\right) \mathrm{d} M_{t}^{-}
$$

The market maker's optimisation problem is

$$
H(t, x, S, \alpha, q)=\sup _{\nu \in \mathcal{A}} \mathbb{E}_{t, x, S, \alpha, q}\left[X_{T}^{\nu}+Q_{T}^{\nu}\left(S_{T}^{\nu}-\operatorname{sign}\left(Q_{T}^{\nu}\right) \Upsilon_{M O}-\psi Q_{T}^{\nu}\right)-\phi \int_{t}^{T}\left(Q_{s}^{\nu}\right)^{2} \mathrm{~d} s\right]
$$

where $\mathbb{E}_{t, x, S, \alpha, q}[\cdot]$ is the expectation operator conditional on $X_{t-}^{\nu}=x, S_{t-}^{\nu}=S, \alpha_{t-}^{\nu}=\alpha, Q_{t-}^{\nu}=$ $q$, and $\mathcal{A}$ is the set of admissible strategies which consists of $\mathcal{F}_{t^{-}}$stopping times $\boldsymbol{\tau}^{ \pm}$and $\mathcal{F}_{t^{-}}$ predictable stochastic control processes $l^{ \pm}$so that $Q_{t}^{\nu} \in[-\bar{Q}, \bar{Q}]$. We give a brief interpretation of each term in the market maker's value function. The terminal cash of the market maker is $X_{T}^{\nu}$. The term $Q_{T}^{\nu}\left(S_{T}^{\nu}-\operatorname{sign}\left(Q_{T}^{\nu}\right) \Upsilon_{M O}-\psi Q_{T}^{\nu}\right)$ represents the earnings or costs from employing MOs to liquidate any outstanding position at the end of the trading horizon, the parameter $\psi$ is a positive constant and $\psi Q_{T}^{\nu}$ represents the extra costs of walking the LOB. The term $\phi \int_{t}^{T}\left(Q_{s}^{\nu}\right)^{2} \mathrm{~d} s$, with $\phi \geq 0$, is the running inventory penalty. The penalty parameter $\phi$ and the inventory cap $\bar{Q}$ represent the market maker's tolerance to inventory risk; see Cartea et al. (2015) and Guéant (2016). Another interpretation of the running inventory penalty is that the market maker is ambiguity averse to the drift of the midprice; see Cartea et al. (2017).

By standard results (see Øksendal and Sulem (2007)) the value function (7) is the unique viscosity solution of the Hamilton-Jacobi-Bellman quasi-variational inequality (HJBQVI)

$$
\begin{array}{r}
\max \left\{\begin{array}{r}
\partial_{t} H+\left(\alpha^{+}+\theta\right)(H(t, x, S+\sigma, \alpha, q)-H) \\
+\left(\alpha^{-}+\theta\right)(H(t, x, S-\sigma, \alpha, q)-H)-\kappa \alpha \partial_{\alpha} H+\frac{1}{2} \xi^{2} \partial_{\alpha \alpha} H-\phi q^{2} \\
+\lambda^{+} \sup _{l^{+} \in\{0,1\}}\left[l^{+}\left(H\left(t, x+\left(S+\Upsilon_{L O}\right), S, \alpha+\eta^{+}, q-1\right)-H\right)\right. \\
\left.+\left(1-l^{+}\right)\left(H\left(t, x, S, \alpha+\eta^{+}, q\right)-H\right)\right] \\
+\lambda^{-} \sup _{l^{-} \in\{0,1\}}\left[l^{-}\left(H\left(t, x-\left(S-\Upsilon_{L O}\right), S, \alpha-\eta^{-}, q+1\right)-H\right)\right. \\
\left.+\left(1-l^{-}\right)\left(H\left(t, x, S, \alpha-\eta^{-}, q\right)-H\right)\right] \\
H\left(t, x+\left(S-\Upsilon_{M O}\right), S, \alpha, q-1\right)-H ; \\
\left.H\left(t, x-\left(S+\Upsilon_{M O}\right), S, \alpha, q+1\right)-H\right\}=0
\end{array}\right.
\end{array}
$$


with terminal condition

$$
H(T, x, S, \alpha, q)=x+q\left(S-\operatorname{sign}(q) \Upsilon_{M O}-\psi q\right)
$$

The stochastic controls, in feedback form, to post LOs are

$$
\begin{array}{ll}
l^{+}=\mathbb{1}_{\left\{H\left(t, x+\left(S+\Upsilon_{L O}\right), S, \alpha+\eta^{+}, q-1\right)>H\left(t, x, S, \alpha+\eta^{+}, q\right)\right\}}, & \text { sell LO } \\
l^{-}=\mathbb{1}_{\left\{H\left(t, x-\left(S-\Upsilon_{L O}\right), S, \alpha-\eta^{-}, q+1\right)>H\left(t, x, S, \alpha-\eta^{-}, q\right)\right\}}, & \text { buy LO } .
\end{array}
$$

In the expression for the control of sell LOs, the term $H\left(t, x+\left(S+\Upsilon_{L O}\right), S, \alpha+\eta^{+}, q-1\right)$ is the value function when the market maker's sell LO is filled by a buy MO. The term $H(t, x, S, \alpha+$ $\left.\eta^{+}, q\right)$ is the value function when a buy MO arrives, but the market maker does not post a sell LO. Similarly, in the expression for the control of buy LOs, the term $H\left(t, x-\left(S-\Upsilon_{L O}\right), S, \alpha-\right.$ $\left.\eta^{-}, q+1\right)$ is the value function when the market maker's buy LO is filled by a sell MO. The term $H\left(t, x, S, \alpha-\eta^{-}, q\right)$ is the value function when a buy MO arrives, but the market maker did not post a buy LO.

Substitute the ansatz $H(t, x, S, \alpha, q)=x+q S+\widetilde{h}(t, \alpha, q)$ in (8) and write

$$
\begin{aligned}
\max \left\{\partial_{t} \widetilde{h}+\alpha \sigma q-\kappa \alpha \partial_{\alpha} \widetilde{h}+\frac{1}{2} \xi^{2} \partial_{\alpha \alpha} \widetilde{h}-\phi q^{2}\right. & \\
& +\lambda^{+} \sup _{l^{+} \in\{0,1\}}\left[l^{+}\left(\Upsilon_{L O}+\widetilde{h}\left(t, \alpha+\eta^{+}, q-1\right)-\widetilde{h}\right)+\left(1-l^{+}\right)\left(\widetilde{h}\left(t, \alpha+\eta^{+}, q\right)-\widetilde{h}\right)\right] \\
& +\lambda^{-} \sup _{l^{-} \in\{0,1\}}\left[l^{-}\left(\Upsilon_{L O}+\widetilde{h}\left(t, \alpha-\eta^{-}, q+1\right)-\widetilde{h}\right)+\left(1-l^{-}\right)\left(\widetilde{h}\left(t, \alpha-\eta^{-}, q\right)-\widetilde{h}\right)\right] \\
& -\Upsilon_{M O}+\widetilde{h}(t, \alpha, q-1)-\widetilde{h} \\
& \left.-\Upsilon_{M O}+\widetilde{h}(t, \alpha, q+1)-\widetilde{h}\right\}=0
\end{aligned}
$$

with terminal condition

$$
\widetilde{h}(T, \alpha, q)=q\left(-\operatorname{sign}(q) \Upsilon_{M O}-\psi q\right)
$$

The optimal feedback controls to post LOs become

$$
l^{+}=\mathbb{1}_{\left\{\Upsilon_{L O}+\widetilde{h}\left(t, \alpha+\eta^{+}, q-1\right)>\widetilde{h}\left(t, \alpha+\eta^{+}, q\right)\right\}} \quad \text { and } \quad l^{-}=\mathbb{1}_{\left\{\Upsilon_{L O}+\widetilde{h}\left(t, \alpha-\eta^{-}, q+1\right)>\widetilde{h}\left(t, \alpha-\eta^{-}, q\right)\right\}}
$$


The three terms of the ansatz represent: the cash accumulated by the strategy up until time $t$, the mark-to-market value of the inventory, and the function $\tilde{h}$ is the extra value the market maker obtains from making markets optimally over the remaining life of the strategy.

Theorem 1. (Verification) Let $\widetilde{h}$ be a solution to 11 and define a candidate solution $\tilde{H}=$ $x+q S+\widetilde{h}(t, \alpha, q)$. Then $\tilde{H}$ equals the value function as defined in (7).

Proof. We follow Øksendal and Sulem (2007). Let the function $f(q)=-\phi q^{2}$. We define the operators $\mathfrak{L}^{\nu}$ and $\mathfrak{M}$ such that the HJBQVI (11) is represented by the form of

$$
\max \left\{\sup _{c \in \mathcal{U}}\left[\mathfrak{L}^{\nu} \widetilde{h}+f\right], \mathfrak{M} \widetilde{h}-\widetilde{h}\right\}=0 .
$$

Let $\widetilde{h}$ be the solution to (11) and define the candidate solution $\tilde{H}=x+q S+\widetilde{h}(t, \alpha, q)$. We want to show that $\tilde{H}=H$.

For any control $\nu=\left(l^{ \pm}, \boldsymbol{\tau}\right)$, where $\boldsymbol{\tau}$ is the union vector of $\boldsymbol{\tau}^{ \pm}$in increasing order, from Itô's Lemma we have

$$
\begin{aligned}
& \mathbb{E}\left[\tilde{H}\left(\tau_{n}^{-}, X_{\tau_{n}^{-}}^{\nu}, S_{\tau_{n}^{-}}^{\nu}, \alpha_{\tau_{n}^{-}}^{\nu}, Q_{\tau_{n}^{-}}^{\nu}\right) \mid \mathcal{F}_{t}\right]-\mathbb{E}\left[\tilde{H}\left(\tau_{n-1}, X_{\tau_{n-1}}^{\nu}, S_{\tau_{n-1}}^{\nu}, \alpha_{\tau_{n-1}}^{\nu}, Q_{\tau_{n-1}}^{\nu}\right) \mid \mathcal{F}_{t}\right] \\
& =\mathbb{E}\left[\int_{\tau_{n-1}}^{\tau_{n}} \mathfrak{L}^{\nu} \widetilde{h}\left(u, \alpha_{u}^{\nu}, Q_{u}^{\nu}\right) \mathrm{d} u \mid \mathcal{F}_{t}\right]
\end{aligned}
$$

and

$$
\begin{aligned}
& \tilde{H}\left(\tau_{n}, X_{\tau_{n}}^{\nu}, S_{\tau_{n}}^{\nu}, \alpha_{\tau_{n}}^{\nu}, Q_{\tau_{n}}^{\nu}\right)-\tilde{H}\left(\tau_{n}^{-}, X_{\tau_{n}^{-}}^{\nu}, S_{\tau_{n}^{-}}^{\nu}, \alpha_{\tau_{n}^{-}}^{\nu}, Q_{\tau_{n}^{-}}^{\nu}\right) \\
& =\mathfrak{M} \widetilde{h}\left(\tau_{n}^{-}, \alpha_{\tau_{n}^{-}}^{\nu}, Q_{\tau_{n}^{-}}^{\nu}\right)-\widetilde{h}\left(\tau_{n}^{-}, \alpha_{\tau_{n}^{-}}^{\nu}, Q_{\tau_{n}^{-}}^{\nu}\right)
\end{aligned}
$$

where, with a slight abuse of notation, we only include the jumps from the control. Summing over $[t, T]$, taking an expectation conditional on $\mathcal{F}_{t}$ and rearranging yields 


$$
\begin{aligned}
\tilde{H}(t, x, S, \alpha, q)= & \mathbb{E}\left[\tilde{H}\left(T, X_{T}^{\nu}, S_{T}^{\nu}, \alpha_{T}^{\nu}, Q_{T}^{\nu}\right) \mid \mathcal{F}_{t}\right] \\
& -\mathbb{E}\left[\int_{t}^{T} \mathfrak{L}^{\nu} \widetilde{h}\left(u, \alpha_{u}^{\nu}, Q_{u}^{\nu}\right) \mathrm{d} u \mid \mathcal{F}_{t}\right] \\
& -\mathbb{E}\left[\sum_{\tau_{n} \leq T} \mathfrak{M} \widetilde{h}\left(\tau_{n}^{-}, \alpha_{\tau_{n}^{-}}^{\nu}, Q_{\tau_{n}^{-}}^{\nu}\right)-\widetilde{h}\left(\tau_{n}^{-}, \alpha_{\tau_{n}^{-}}^{\nu}, Q_{\tau_{n}^{-}}^{\nu}\right) \mid \mathcal{F}_{t}\right] .
\end{aligned}
$$

From the HJBQVI in 11 we have

$$
\begin{aligned}
\tilde{H}(t, x, S, \alpha, q) & \geq \mathbb{E}\left[\tilde{H}\left(T, X_{T}^{\nu}, S_{T}^{\nu}, \alpha_{T}^{\nu}, Q_{T}^{\nu}\right)-\phi \int_{t}^{T}\left(Q_{u}^{\nu}\right)^{2} \mathrm{~d} u \mid \mathcal{F}_{t}\right] \\
& =\mathbb{E}\left[X_{T}^{\nu}+Q_{T}^{\nu}\left(S_{T}^{\nu}-\operatorname{sign}\left(Q_{T}^{\nu}\right) \Upsilon_{M O}-\psi Q_{T}^{\nu}\right)-\phi \int_{t}^{T}\left(Q_{u}^{\nu}\right)^{2} \mathrm{~d} u \mid \mathcal{F}_{t}\right]
\end{aligned}
$$

The inequality above holds for any control $\nu$, thus we have

$$
\begin{aligned}
\tilde{H}(t, x, S, \alpha, q) & \geq \sup _{\boldsymbol{\nu} \in \mathcal{A}} \mathbb{E}_{t, x, S, \alpha, q}\left[X_{T}^{\nu}+Q_{T}^{\nu}\left(S_{T}^{\nu}-\operatorname{sign}\left(Q_{T}^{\nu}\right) \Upsilon_{M O}-\psi Q_{T}^{\nu}\right)-\phi \int_{t}^{T}\left(Q_{u}^{\nu}\right)^{2} \mathrm{~d} u\right] \\
& =H(t, x, S, \alpha, q) .
\end{aligned}
$$

Now if we use the optimal control $\nu^{*},(19)$ becomes

$$
\begin{aligned}
\tilde{H}(t, x, S, \alpha, q) & =\mathbb{E}\left[\tilde{H}\left(T, X_{T}^{\nu^{*}}, S_{T}^{\nu^{*}}, \alpha_{T}^{\nu^{*}}, Q_{T}^{\nu^{*}}\right)-\phi \int_{t}^{T}\left(Q_{u}^{\nu^{*}}\right)^{2} \mathrm{~d} u \mid \mathcal{F}_{t}\right] \\
& =\mathbb{E}\left[X_{T}^{\nu^{*}}+Q_{T}^{\nu}\left(S_{T}^{\nu^{*}}-\operatorname{sign}\left(Q_{T}^{\nu^{*}}\right) \Upsilon_{M O}-\psi Q_{T}^{\nu^{*}}\right)-\phi \int_{t}^{T}\left(Q_{u}^{\nu^{*}}\right)^{2} \mathrm{~d} u \mid \mathcal{F}_{t}\right] \\
& \leq \sup _{\boldsymbol{\nu} \in \mathcal{A}} \mathbb{E}_{t, x, S, \alpha, q}\left[X_{T}^{\nu}+Q_{T}^{\nu}\left(S_{T}^{\nu}-\operatorname{sign}\left(Q_{T}^{\nu}\right) \Upsilon_{M O}-\psi Q_{T}^{\nu}\right)-\phi \int_{t}^{T}\left(Q_{u}^{\nu}\right)^{2} \mathrm{~d} u\right] \\
& =H(t, x, S, \alpha, q) .
\end{aligned}
$$

Hence $H=\hat{H}$.

Finally, we note that in our model the jumps in the midprice are always of size one tick. One could also assume that $\sigma$ in (1) is the average number of ticks the midprice jumps when there is a price innovation. Also, one could consider a model where the spread is also stochastic; see Cartea et al. (2018). For simplicity, we assume the spread is constant at one tick, which is the 
case of the large-stick stocks such as the ones we study here.

\subsection{Parameter estimates}

In this section we employ Nasdaq high-frequency data to estimate some of the model parameters. The data are messages sent to the Nasdaq exchange, with nanosecond time stamps, that indicate the events in the LOB such as arrival and cancellation of LOs, and arrival of MOs. For simplicity, we assume that the volatility parameter $\xi$ in $(4)$ is zero.

For the time interval $[0, T]$, let

$$
t^{+}=\left\{t_{1}^{+}, t_{2}^{+}, \ldots, t_{m^{+}}^{+}\right\} \quad \text { and } \quad t^{-}=\left\{t_{1}^{-}, t_{2}^{-}, \ldots, t_{m^{-}}^{-}\right\}
$$

denote the times when the midprice jumps, up $(+)$ and down $(-)$, and let the arrays

$$
\tau^{0+}=\left\{\tau_{1}^{0+}, \tau_{2}^{0+}, \ldots, \tau_{n^{+}}^{0+}\right\} \quad \text { and } \quad \tau^{0-}=\left\{\tau_{1}^{0-}, \tau_{2}^{0-}, \ldots, \tau_{n^{-}}^{0-}\right\}
$$

represent the arrival times of the MOs: the superscripts $0+$ and 0 - denote buy and sell orders, respectively.

We also denote by $\tau^{0}$ the array that combines $\tau^{0+}, \tau^{0-}$ and $\{0, T\}$, such that $\tau^{0}$ is an increasing

sequence that starts at $\tau_{0}^{0}=0$ and ends at time $\tau_{n^{+}+n^{-}+1}^{0}=T$. The entries of the array $\tau^{0}$ are observed, so we write the alpha signal as follows:

$$
\alpha_{t}=\eta^{+} \sum_{i=1}^{n^{+}} \exp \left(-\kappa\left(t-\tau_{i}^{0+}\right)\right) \mathbb{1}_{\left\{t \geq \tau_{i}^{0+}\right\}}-\eta^{-} \sum_{i=1}^{n^{-}} \exp \left(-\kappa\left(t-\tau_{i}^{0-}\right)\right) \mathbb{1}_{\left\{t \geq \tau_{i}^{0-}\right\}}
$$

where $\mathbb{1}_{\{\cdot\}}$ is the indicator function. 
The log-likelihood of $t^{ \pm}$, given $\tau^{0 \pm}$, is

$$
\begin{aligned}
& \mathscr{L}(\Theta) \\
& =\log \mathbb{P}\left(t^{ \pm} \mid \tau^{0 \pm}, \Theta\right) \\
& =\log \mathbb{P}\left(t^{+} \mid \tau^{0 \pm}, \Theta\right)+\log \mathbb{P}\left(t^{-} \mid \tau^{0 \pm}, \Theta\right) \\
& =\log \left[e^{-\int_{0}^{T} \mu_{s}^{+} \mathrm{d} s} \prod_{i=1}^{m^{+}} \mu_{t_{i}^{+}}^{+}\right]+\log \left[e^{-\int_{0}^{T} \mu_{s}^{-} \mathrm{d} s} \prod_{i=1}^{m^{-}} \mu_{t_{i}^{-}}^{-}\right] \\
& =-\int_{0}^{T} \mu_{s}^{+} \mathrm{d} s+\sum_{i=1}^{m^{+}} \log \mu_{t_{i}^{+}}^{+}-\int_{0}^{m^{-}} \mu_{s}^{-} \mathrm{d} s+\sum_{i=1}^{T} \log \mu_{t_{i}^{-}}^{-} \\
& =-2 \theta T-\int_{0}^{T}\left(\left(\alpha_{s}\right)_{+}-\left(\alpha_{s}\right)_{-}\right) \mathrm{d} s+\sum_{i=1}^{m^{+}} \log \left[\left(\alpha_{t_{i}^{+}}\right)_{+}+\theta\right]+\sum_{i=1}^{m^{-}} \log \left[\left(\alpha_{t_{i}^{-}}\right)_{-}+\theta\right]
\end{aligned}
$$

where $\Theta=\left(\kappa, \eta^{+}, \eta^{-}, \theta\right)$ denotes the set of parameters to estimate.

Use (25) to write each term in the log-likelihood. We start with the term $\alpha_{t^{-}}$, which is given by

$$
\alpha_{t^{-}}=\eta^{+} \sum_{i=1}^{n^{+}} e^{-\kappa\left(t-\tau_{i}^{0+}\right)} \mathbb{1}_{\left\{t>\tau_{i}^{0+}\right\}}-\eta^{-} \sum_{i=1}^{n^{-}} e^{-\kappa\left(t-\tau_{i}^{0-}\right)} \mathbb{1}_{\left\{t>\tau_{i}^{0-}\right\}}
$$

Now, to write the integrated alpha signal that appears in the last line of $(26)$ we note that the process $\alpha_{t}$ does not change sign in the interval $\left[\tau_{i}^{0}, \tau_{i+1}^{0}\right)$ for $i=0,1, \ldots, n^{+}+n^{-}$, so by the definition of $\alpha_{t}$ we have

$$
\begin{aligned}
\int_{0}^{T}\left(\alpha_{s}\right)_{+} \mathrm{d} s=-\frac{1}{\kappa} \sum_{i=0}^{n^{+}+n^{-}} \mathbb{1}_{\left\{\alpha_{\tau_{i}^{0}} \geq 0\right\}} & {\left[\eta^{+} \sum_{j=1}^{n+}\left(e^{-\kappa\left(\tau_{i+1}^{0} \vee \tau_{j}^{0+}-\tau_{j}^{0+}\right)}-e^{-\kappa\left(\tau_{i}^{0} \vee \tau_{j}^{0+}-\tau_{j}^{0+}\right)}\right)\right.} \\
& \left.-\eta^{-} \sum_{j=1}^{n-}\left(e^{-\kappa\left(\tau_{i+1}^{0} \vee \tau_{j}^{0-}-\tau_{j}^{0-}\right)}-e^{-\kappa\left(\tau_{i}^{0} \vee \tau_{j}^{0-}-\tau_{j}^{0-}\right)}\right)\right],
\end{aligned}
$$


and

$$
\begin{aligned}
\int_{0}^{T}\left(\alpha_{s}\right)_{-} \mathrm{d} s=\frac{1}{\kappa} \sum_{i=0}^{n^{+}+n^{-}} \mathbb{1}_{\left\{\alpha_{\tau_{i}^{0}} \leq 0\right\}}\left[\eta^{+} \sum_{j=1}^{n+}\left(e^{-\kappa\left(\tau_{i+1}^{0} \vee \tau_{j}^{0+}-\tau_{j}^{0+}\right)}-e^{-\kappa\left(\tau_{i}^{0} \vee \tau_{j}^{0+}-\tau_{j}^{0+}\right)}\right)\right. \\
\left.-\eta^{-} \sum_{j=1}^{n-}\left(e^{-\kappa\left(\tau_{i+1}^{0} \vee \tau_{j}^{0-}-\tau_{j}^{0-}\right)}-e^{-\kappa\left(\tau_{i}^{0} \vee \tau_{j}^{0-}-\tau_{j}^{0-}\right)}\right)\right] .
\end{aligned}
$$

Finally, maximise the log-likelihood to obtain the parameter estimates:

$$
\widehat{\Theta}=\operatorname{argmax} \mathscr{L}(\Theta)
$$

Table 1 reports parameter estimates for 10 stocks traded in Nasdaq - we employ data from 10:00 to 15:30 on 11 February 2019. From Table 1 we observe that the size of the jumps in the alpha signal induced by the arrival of MOs is much larger than the baseline of the intensities for midprice jumps, i.e., $\hat{\eta}^{ \pm} \gg \hat{\theta}$. The value of the mean-reverting speed of the alpha process, $\hat{\kappa}$, is at least as large as the size of the jumps in the alpha process, and for some stocks it is much larger the size of the jumps. We recall the half-life of $\alpha$ is $\kappa^{-1} \log 2$, and from Table 1 we see that the estimated half-lives are all less than 0.02 seconds for the stocks we study. This shows that the alpha signal decays very quickly, so the upward (downward) momentum of the midprice lasts for a very short period of time. Only market participants (e.g., high-frequency traders and high-frequency market makers) who are able to process information and access the market within milliseconds will be able to take advantage of the alpha signal; see Cartea and Sánchez-Betancourt (2018) for a discussion of latency in electronic markets.

\begin{tabular}{c|cccccc}
\hline \hline & $\hat{\kappa}$ & $\hat{\eta}^{+}$ & $\hat{\eta}^{-}$ & $\hat{\theta}$ & $\hat{\lambda}^{+}$ & $\hat{\lambda}^{-}$ \\
\hline COST & 85.669 & 92.024 & 78.548 & 0.446 & 0.074 & 0.086 \\
\hline CSCO & 310.790 & 75.263 & 52.646 & 0.055 & 0.086 & 0.108 \\
\hline EBAY & 267.880 & 30.221 & 54.676 & 0.046 & 0.129 & 0.054 \\
\hline EXPE & 62.425 & 54.592 & 49.350 & 0.342 & 0.084 & 0.099 \\
\hline GILD & 269.255 & 118.323 & 105.776 & 0.383 & 0.101 & 0.105 \\
\hline MSFT & 225.456 & 97.085 & 84.773 & 0.575 & 0.287 & 0.268 \\
\hline ORCL & 355.617 & 105.237 & 77.118 & 0.091 & 0.072 & 0.061 \\
\hline PYPL & 236.140 & 134.227 & 106.058 & 0.519 & 0.148 & 0.156 \\
\hline QCOM & 459.756 & 104.341 & 93.905 & 0.146 & 0.122 & 0.125 \\
\hline VRTX & 46.754 & 26.092 & 35.014 & 0.118 & 0.022 & 0.027 \\
\hline \hline
\end{tabular}

Table 1: Parameter estimates. Data: Nasdaq, from 10:00 to 15:30, 11 February 2019. 


\begin{tabular}{c|cccc}
\hline \hline & $\hat{\kappa}$ & $\hat{\eta}$ & $\hat{\theta}$ & $\hat{\lambda}$ \\
\hline COST & 83.622 & 82.638 & 0.446 & 0.080 \\
\hline CSCO & 309.208 & 62.119 & 0.055 & 0.097 \\
\hline EBAY & 270.145 & 37.881 & 0.046 & 0.092 \\
\hline EXPE & 66.551 & 54.665 & 0.343 & 0.092 \\
\hline GILD & 265.501 & 109.970 & 0.383 & 0.103 \\
\hline MSFT & 238.284 & 95.052 & 0.576 & 0.278 \\
\hline ORCL & 370.179 & 95.664 & 0.091 & 0.066 \\
\hline PYPL & 254.785 & 126.808 & 0.521 & 0.152 \\
\hline QCOM & 441.456 & 95.253 & 0.146 & 0.124 \\
\hline VRTX & 45.930 & 30.428 & 0.118 & 0.025 \\
\hline \hline
\end{tabular}

Table 2: Parameter estimates with constraints of symmetry. Data: Nasdaq, from 10:00 to 15:30, 11 February 2019.

\subsection{Optimal strategy}

In this section we solve the market maker's HJBQVI numerically, where we employ the parameters in Table 3, and discuss the stylized features of the optimal strategy. Note that for the large-tick stocks we study, the quoted spread is very often one tick, i.e, $2 \Delta=0.01$, which is also the size of the price innovations in our model.

\begin{tabular}{cccccccccccccc}
\hline \hline$\Delta$ & $\varepsilon_{M O}$ & $\varepsilon_{L O}$ & $\sigma$ & $\theta$ & $\eta$ & $\kappa$ & $\xi$ & $\lambda^{+}$ & $\lambda^{-}$ & $\phi$ & $\psi$ & $T$ & $\bar{Q}$ \\
\hline 0.005 & 0.003 & 0.002 & 0.01 & 0.1 & 60 & 200 & 1 & 1 & 1 & $10^{-6}$ & 0 & 60 & 4 \\
\hline \hline
\end{tabular}

Table 3: Model parameters.

Figure 1 shows the market maker's optimal strategy for the last ten seconds of the trading horizon, i.e., $t \in[50,60]$, for three inventory levels (far from maturity, the optimal strategy does not vary much as time elapses, so we omit the first 50 seconds). We first focus on the case $q=0$. When the value of $\alpha_{t}$ is near zero the strategy posts both sell and buy LOs. As the value of $\alpha$ increases, the strategy posts buy LOs because the midprice of the asset will, on average, increase due to the positive trend. The strategy does not post sell LOs at the best ask price because the LOs would be picked off by high-frequency traders who also trade on a momentum signal. As the value of $\alpha$ increases further, the market maker sends buy MOs in anticipation of a price increase, i.e., due to the strong upward pressure on the midprice of the asset. The optimal strategy is similar when the value of $\alpha$ is negative, and in this particular case the optimal strategy is symmetric with respect to $\alpha=0$ because the parameters we choose are symmetric, i.e., $\lambda^{+}=\lambda^{-}$and $q=0$. Near maturity, the regions of LOs and MOs shrink. We notice there is a sliver near maturity where the optimal strategy does not post LOs or send MOs. In this region, the market maker's position is already zero when it is close to maturity 
and the expected profit from taking positions to earn the spread are offset by the uncertainty in the midprice and liquidation costs. Near maturity, if the non-zero inventory is not cleared, the market maker needs to pay the spread and fees to unwind inventories with MOs.

If the inventory of the market maker is short, for example $q=-1$, the buy LO region and the buy MO region expand compared with that of the strategy for $q=0$, and the sell LO and the sell MO regions shrink. The optimal strategy for $q=1$ is symmetric with the optimal strategy when $q=-1$ because of the symmetry in the parameters.
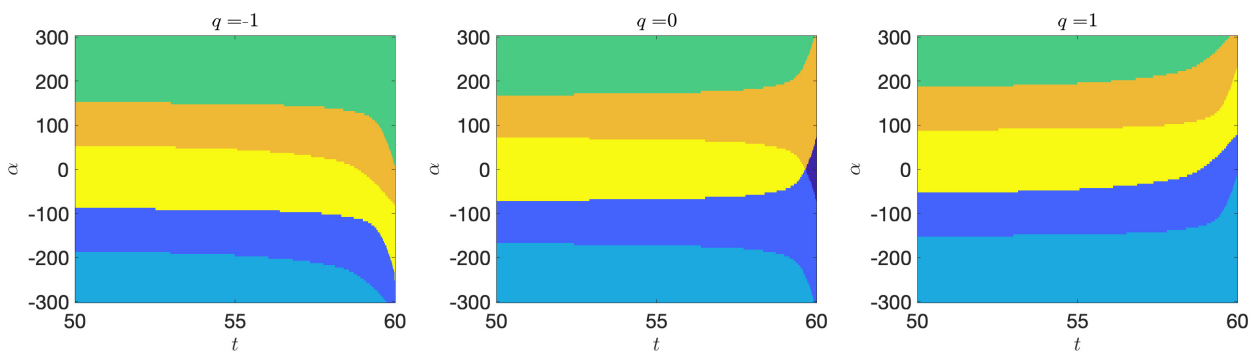

Figure 1: Optimal strategy of the market maker. Dark blue: no LOs in the LOB and no MOs. Blue: post sell LO $\left(l^{+}=1\right)$. Light blue: send sell MO $\left(\boldsymbol{\tau}^{-}\right)$. Orange: post buy LO $\left(l^{-}=1\right)$. Green: send buy MO $\left(\boldsymbol{\tau}^{+}\right)$. Yellow: post both sell and buy LO $\left(l^{+}=l^{-}=1\right)$. Near maturity, there is a green sliver when $q=-1$, a dark blue sliver when $q=0$, and a light blue sliver when $q=1$.

We recall that the value function is $H=x+q S+\widetilde{h}(t, \alpha, q)$. Figure 2 shows the surface of the function $\widetilde{h}$ evaluated at $t=0$. Recall the function $\tilde{h}$ is the extra value the market maker obtains from making markets optimally over the remaining life of the strategy. The value of $\widetilde{h}$ is lowest when both the initial inventory is positive and the initial value of alpha signal is negative or when the initial inventory is negative and the alpha signal is positive. Note that if the market maker has a large long position in the asset and its midprice is expected to trend downwards, the value of the inventory is expected to decrease. Similarly, the value of $\widetilde{h}$ is highest when the initial inventory is in the direction of the initial alpha signal (i.e., the value of $\alpha$ is positive and $q$ is positive or the value of $\alpha$ is negative and $q$ is negative) so the value of the inventory is expected to appreciate.

\section{Simulation and performance of strategy.}

\subsection{Sample paths}

Figure 3 shows one simulation of the strategy: midprice sample path and trading activity, alpha signal, and inventory path. We observe that the market maker trades mostly using LOs. When there is a steep change in the midprice the market maker executes MOs to adjust their inventory 


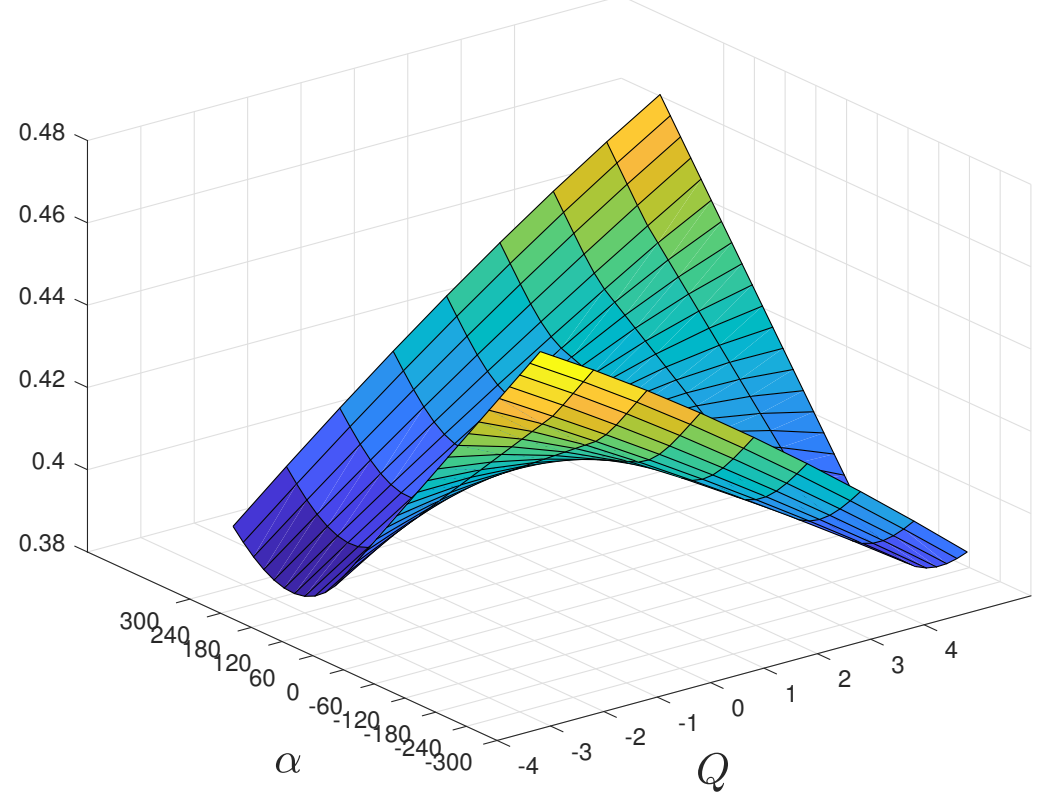

Figure 2: Surface of function $\tilde{h}$. The value of $\tilde{h}$ is highest when the value of inventory is expected to appreciate and lowest when the value of inventory is expected to depreciate.

and if the alpha signal indicates an imminent change in prices the strategy also sends MOs. The strategy employs the alpha signal to keep the inventory on target, minimise adverse selection costs, and to execute speculative directional MOs. During most of the trading horizon, the strategy employs either two-sided LOs or one-sided LOs and employs only on MO at the end of the trading horizon. When the alpha signal is positive (negative), the strategy tends not to post sell (buy) LOs to avoid adverse selection costs. Near the end of the trading window we observe an upward spike in the alpha signal that triggers a buy $\mathrm{MO}$, which is also required to unwind inventories because the strategy is close to expiry and the value of the inventory position is short, see panel (c). Throughout the trading horizon, the market maker's inventory $Q_{t}^{\nu}$ tends to revert toward zero because the strategy benefits from executing roundtrip trades and accounts for the market maker's tolerance to inventory risk. Notice that panel (b) shows the time of the alpha signal being away from zero is much shorter compared to the time of the alpha signal being practically zero. For the parameters we choose as in Table 3 , the half life of the alpha signal is approximately 0.02 seconds. This makes the actual implementation of the strategy difficult as the market maker need to react instantaneously, which is challenging in terms of both software and hardware. 


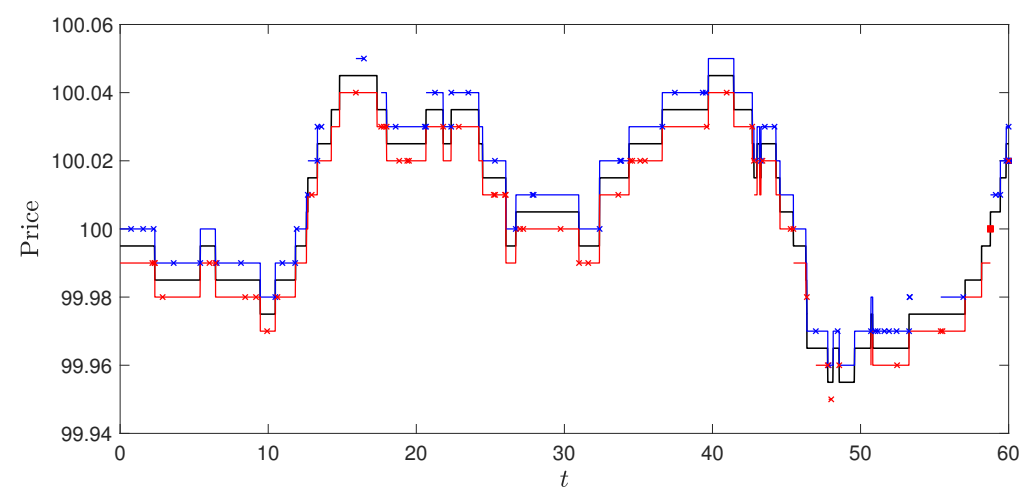

(a) Black line: midprice. Blue line: post sell LO. Blue cross: sell LO is filled. Red line: post buy LO. Red cross: buy LO is filled. Blue square: execute sell MO. Red square: execute buy MO.

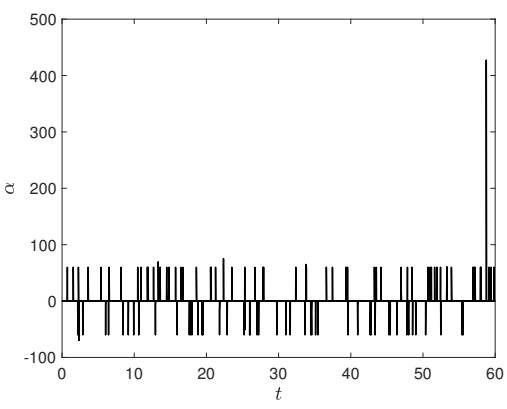

(b) Alpha signal

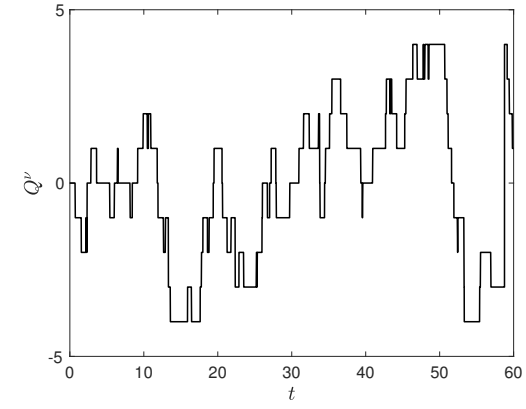

(c) Inventory

Figure 3: One simulation: sample path of midprice, alpha signal and inventory.

\subsection{Distribution of orders traded}

Figure 4 shows the distribution (10,000 simulations) of the orders traded for various values of the inventory penalty parameter $\phi$. For example, as the value of the parameter $\phi$ decreases, both the number of filled LOs and the number of executed MOs increase because the market maker is more willing to take positions with LOs and to employ more MOs to adjust their inventory.

In the figures, light green corresponds to $\phi=10^{-6}$, red corresponds to $\phi=5 \times 10^{-4}$, and blue corresponds to $\phi=10^{-3}$. In panels (c) and (d) the superposition of up to three colours appears in different shades of green. Note that when the inventory penalty parameter $\phi$ is smallest, there are simulation runs in which the strategy employs more than two sell and two buy MOs, and for larger values of the parameter $\phi$ the maximum number of sell or buy MOs we observe is two. Clearly, as the market maker becomes more tolerant to bear inventory risk, it is optimal to build larger inventory positions and employ more speculative MOs to take advantage of the alpha signal and to send MOs to keep the inventory on target. 


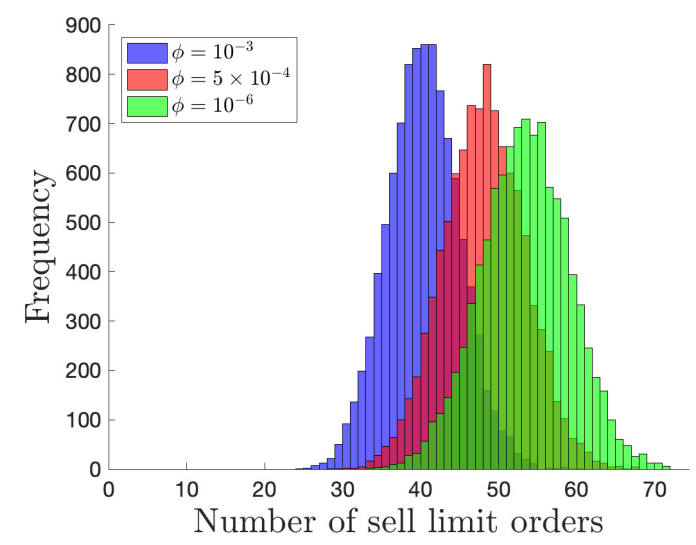

(a) Sell LOs

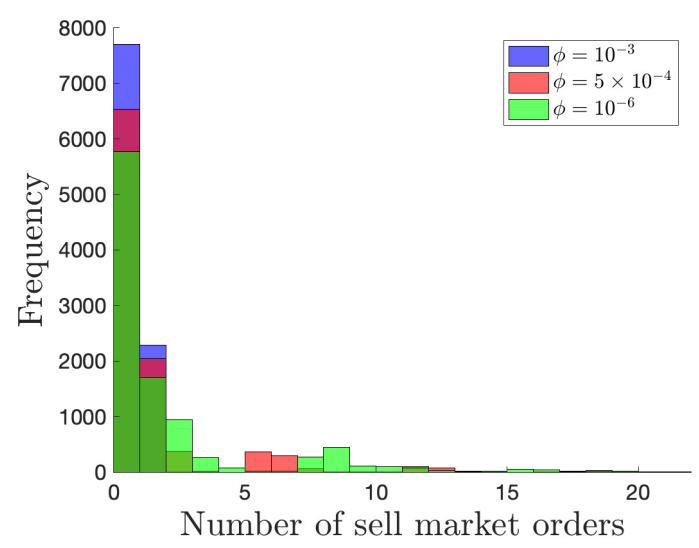

(c) Sell MOs

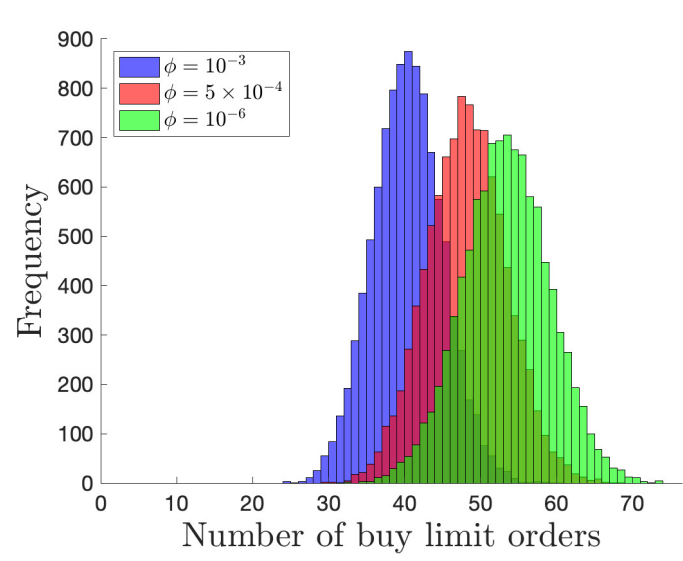

(b) Buy LOs

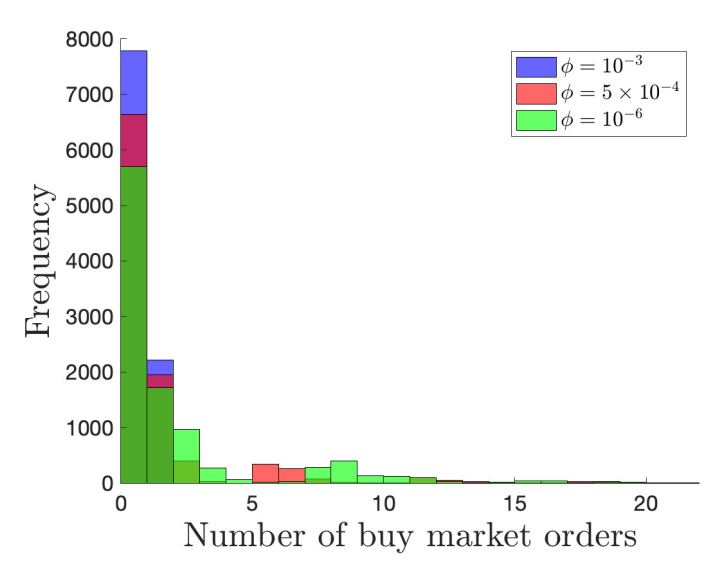

(d) Buy MOs

Figure 4: Distributions of the number of filled LOs and executed MOs for three values of the inventory penalty parameter $\phi$.

\subsection{Tradeoff: mean and standard deviation of $P \mathscr{G} L$}

In this section we examine the mean and the standard deviation of $\mathrm{P} \& \mathrm{~L}$ for various values of the running inventory penalty parameter and the cap on the inventory, both of which represent the market maker's willingness to bear inventory risk. The trading horizon is $T=60$ seconds. We also compare the P\&L of a strategy that incorporates the alpha signal with a strategy that assumes $\alpha_{t}=0$ throughout the trading horizon.

Figure 5 plots the mean of $\mathrm{P} \& \mathrm{~L}$ against the and the standard deviation of $\mathrm{P} \& \mathrm{~L}$ for a range of values of $\phi$ and Figure 6 plots the mean of $\mathrm{P} \& \mathrm{~L}$ against the standard deviation of $\mathrm{P} \& \mathrm{~L}$ for a range of $\bar{Q}$, which is the cap on inventory. Both figures show the results of the optimal strategy with the alpha signal and without employing the alpha signal, i.e, $\alpha_{t}=0$, during the trading horizon. Figure 5 shows that both the mean and the standard deviation of the P\&L increase when the 
value of the inventory penalty parameter $\phi$ decreases. As the inventory penalty becomes less severe, the strategy takes larger positions in the asset (long and short) to benefit from the alpha signal. When the value of the penalty parameter $\phi$ is high, a slight decrease in the value of $\phi$ causes an increase in the mean of the $\mathrm{P} \& \mathrm{~L}$ without a significant increase in the standard deviation of P\&L. Also, the P\&L of the optimal strategy is higher than the P\&L of the strategy of an investor who assumes $\alpha_{t}=0$ for the whole trading horizon. For low values of $\phi$, the difference between the two strategies is about $0.02 \$$ for a trading horizon of 60 seconds.

Figure 6 shows that both the mean and the standard deviation of the P\&L increase when the value of the cap $\bar{Q}$ increases. A larger allowance of maximum and minimum inventory levels leads to higher expected profits because the strategy can perform, on average, more roundtrip trades and because the strategy can take full advantage of the momentum in the price of the asset. For small values of $\bar{Q}$, the mean of the P\&L increases without a significant increase in the standard deviation of the P\&L when $\bar{Q}$ increases. The P\&L with the optimal strategy is higher than the P\&L of a strategy that assumes $\alpha_{t}=0$. For higher values of $\bar{Q}$, the difference in mean P\&Ls is about $0.04 \$$ for a trading horizon of 60 seconds.

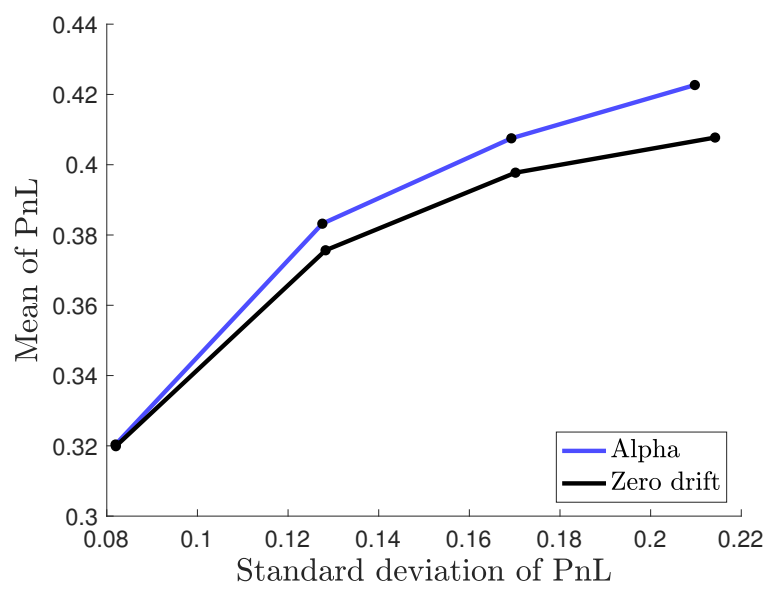

Figure 5: Risk-reward profile with $\bar{Q}=4, T=60$ seconds. From left to right, $\phi=10^{-3}, 5 \times 10^{-4}, 2 \times$ $10^{-4}, 10^{-6}$.

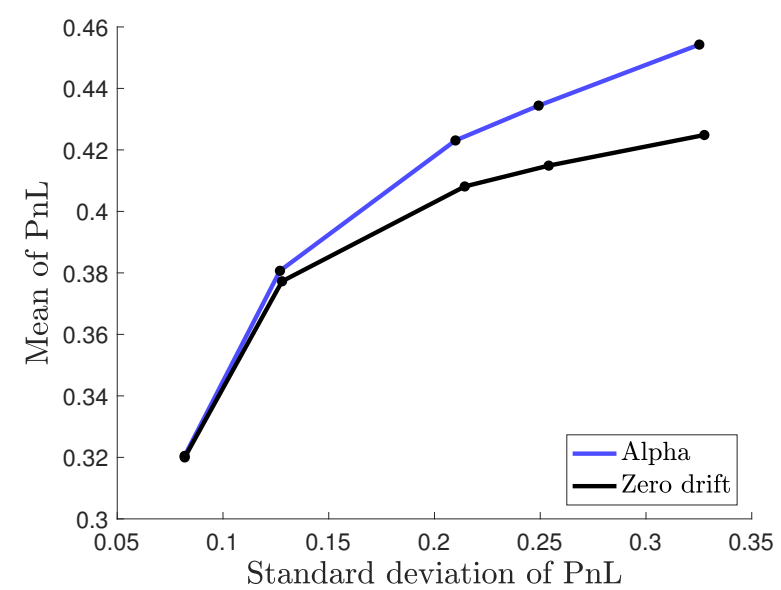

Figure 6: Risk-reward profile with $\phi=10^{-6}, T=$ 60 seconds. From left to right, $\bar{Q}=1,2,4,5,7$.

\section{Simulation results with parameters estimated from Nasdaq data}

We use the parameter estimates of 3 stocks reported in Table 1 and assume $\xi=0$, i.e., there is no diffusion in the alpha signal. We run 10,000 simulations with $T=300$ seconds and other model parameters as in Table 3. Recall that the parameter estimates are obtained from high-frequency Nasdaq data on 11 February 2019 (10:00 to 15:30).

Table 4 reports the mean and the standard deviation of the simulated P\&L for a range of values 
of the running inventory penalty parameter $\phi$. Observe that as the value of the parameter $\phi$ increases, both the mean and the standard deviation of the P\&L decrease.

\begin{tabular}{|c|c|c|c|c|}
\hline \multirow{2}{*}{ Stock } & \multicolumn{4}{|c|}{$\phi$} \\
\hline & $10^{-6}$ & $3 \times 10^{-5}$ & $10^{-4}$ & $3 \times 10^{-4}$ \\
\hline $\mathrm{CSCO}$ & $0.257393(0.170465)$ & $0.228978(0.105016)$ & $0.188893(0.067854)$ & $0.188898(0.067851)$ \\
\hline EBAY & $0.266769(0.158333)$ & $0.240957(0.099900)$ & $0.238051(0.096836)$ & $0.199573(0.065479)$ \\
\hline GILD & $0.152533(0.370212)$ & $0.135067(0.221638)$ & $0.108451(0.138233)$ & $0.108458(0.138176)$ \\
\hline
\end{tabular}

Table 4: Mean, in $\$$, of the P\&L and standard deviation of P\&L in brackets. Parameters as in Table 1 $T=300$ seconds, $\bar{Q}=4$, and various choices of $\phi$.

Table 5 reports the mean and the standard deviation of the simulated P\&L for a range of $\bar{Q}$ with a fixed value of $\phi$. As $\bar{Q}$ increases, both the mean and the standard deviation of P\&L increase.

\begin{tabular}{|c|c|c|c|c|}
\hline \multirow{2}{*}{ Stock } & \multicolumn{4}{|c|}{$\bar{Q}$} \\
\hline & 2 & 4 & 7 & 10 \\
\hline $\mathrm{CSCO}$ & $0.228793(0.105515)$ & $0.257393(0.170465)$ & $0.267399(0.239815)$ & $0.267638(0.250639)$ \\
\hline EBAY & $0.241087(0.100206)$ & $0.266769(0.158333)$ & $0.270478(0.219711)$ & $0.270970(0.229488)$ \\
\hline GILD & $0.135825(0.222287)$ & $0.152533(0.370212)$ & $0.175081(0.538307)$ & $0.175016(0.538375)$ \\
\hline
\end{tabular}

Table 5: Mean, in $\$$, of the P\&L and standard deviation of P\&L in brackets. Parameters as in Table 1 , $T=300$ seconds, $\phi=10^{-6}$, and various choices of $\bar{Q}$.

These results coincide with the properties we found in Section 3.3 .

\section{Numerical scheme}

To solve (11) numerically, we assume the signal $\alpha$ lives on a grid with boundaries $-A \leq \alpha \leq A$ for some constant $A \in(0,+\infty)$, and the additional boundary conditions:

$$
\partial_{\alpha \alpha} \tilde{h}(t,-A, q)=0 \quad \text { and } \quad \partial_{\alpha \alpha} \widetilde{h}(t, A, q)=0
$$

for all $t \in[0, T]$ and $q \in[-\bar{Q}, \bar{Q}] \cap \mathbb{Z}$. For any $\alpha>A$ we use linear extrapolation from $\widetilde{h}(t, A-\delta \alpha, q)$ and $\widetilde{h}(t, A, q)$, and similarly for any $\alpha<-A$. For the simulations presented above we used $A=300$.

To prove the convergence of the numerical scheme we impose bounds on $\alpha$, which also bound the rate of increase or decrease of the midprice. Assume

$$
\mu_{t}^{+}=\left(\bar{\alpha}_{t}\right)_{+}+\theta, \quad \mu_{t}^{-}=\left(\bar{\alpha}_{t}\right)_{-}+\theta
$$


where

$$
\bar{\alpha}_{t}=\min \left\{A, \max \left\{-A, \alpha_{t}\right\}\right\}
$$

for some constant $A \in(0,+\infty)$.

We also impose the same bound on the term $-\kappa \alpha \partial_{\alpha} \widetilde{h}$ in (11). Thus, we solve the HJBQVI:

$$
\begin{aligned}
\max \left\{\partial_{t} h+\bar{\alpha} \sigma q-\kappa \bar{\alpha} \partial_{\alpha} h+\frac{1}{2} \xi^{2} \partial_{\alpha \alpha} h-\phi q^{2}\right. & \\
& +\lambda^{+} \sup _{l^{+} \in\{0,1\}}\left[l^{+}\left(\Upsilon_{L O}+h\left(t, \alpha+\eta^{+}, q-1\right)-h\right)+\left(1-l^{+}\right)\left(h\left(t, \alpha+\eta^{-}, q\right)-h\right)\right] \\
& +\lambda^{-} \sup _{l^{-} \in\{0,1\}}\left[l^{-}\left(\Upsilon_{L O}+h\left(t, \alpha-\eta^{-}, q+1\right)-h\right)+\left(1-l^{-}\right)\left(h\left(t, \alpha-\eta^{-}, q\right)-h\right)\right] \\
& -\Upsilon_{M O}+h(t, \alpha, q-1)-h ; \\
& \left.-\Upsilon_{M O}+h(t, \alpha, q+1)-h\right\}=0
\end{aligned}
$$

with terminal condition

$$
h(T, \alpha, q)=q\left(-\operatorname{sign}(q) \Upsilon_{M O}-\psi q\right)
$$

Theorem 2. (Comparison principle) Let $h_{1}$ and $h_{2}$ be, respectively, a bounded subsolution and a bounded supersolution of (34). Suppose that $h_{1}(T) \leq h_{2}(T)$. Then $h_{1} \leq h_{2}$.

Proof. See Theorem 2.5.11. of Seydel (2010).

Here we describe the numerical scheme we employ to solve (34). Let $\mathbb{T}_{\delta t}$ be the uniform grid on $[0, T]$ with step size $\delta t>0$ and $\mathbb{R}_{\delta \alpha}$ be the uniform grid on $\mathbb{R}$ with step size $\delta \alpha>0$. We define, for any function $\varphi:[0, T] \times \mathbb{R}_{\delta \alpha} \times([-\bar{Q}, \bar{Q}] \cap \mathbb{Z}) \rightarrow \mathbb{R}$, the operator

$$
\mathcal{S}^{\delta t, \delta \alpha}(t, \alpha, q, \varphi)=\max \left[\mathcal{T}^{\delta t, \delta \alpha}(t, \alpha, q, \varphi), \mathcal{M}^{\delta t, \delta \alpha}(t, \alpha, q, \varphi)\right]
$$


where

$$
\begin{aligned}
& \mathcal{T}^{\delta t, \delta \alpha}(t, \alpha, q, \varphi) \\
& =\varphi+\delta t\left\{\bar{\alpha} \sigma q+\kappa \bar{\alpha}_{-} \frac{\varphi(t, \alpha+\delta \alpha, q)-\varphi(t, \alpha, q)}{\delta \alpha}-\kappa \bar{\alpha}_{+} \frac{\varphi(t, \alpha, q)-\varphi(t, \alpha-\delta \alpha, q)}{\delta \alpha}\right. \\
& \quad+\frac{\xi^{2}}{2} \frac{\varphi(t, \alpha+\delta \alpha, q)-2 \varphi(t, \alpha, q)+\varphi(t, \alpha-\delta \alpha, q)}{\delta \alpha^{2}}-\phi q^{2} \\
& +\lambda^{+} \sup _{l^{+} \in\{0,1\}}\left[l^{+}\left(\Upsilon_{L O}+\mathcal{I}^{+} \varphi(t, \alpha, q-1)-\varphi\right)+\left(1-l^{+}\right)\left(\mathcal{I}^{+} \varphi(t, \alpha, q)-\varphi\right)\right] \\
& \left.+\lambda^{-} \sup _{l^{-} \in\{0,1\}}\left[l^{-}\left(\Upsilon_{L O}+\mathcal{I}^{-} \varphi(t, \alpha, q+1)-\varphi\right)+\left(1-l^{-}\right)\left(\mathcal{I}^{-} \varphi(t, \alpha, q)-\varphi\right)\right]\right\},
\end{aligned}
$$

and

$$
\mathcal{M}^{\delta t, \delta \alpha}(t, \alpha, q, \varphi)=\max \left\{\varphi(t, \alpha, q-1)-\Upsilon_{M O}, \quad \varphi(t, \alpha, q+1)-\Upsilon_{M O}\right\}
$$

The linear interpolation operators $\mathcal{I}^{+}$and $\mathcal{I}^{-}$for the jumps in $\alpha$ are given by

$$
\begin{aligned}
\mathcal{I}^{+} \varphi(t, \alpha, q)= & \varphi\left(t, \alpha+\left\lfloor\frac{\eta^{+}}{\delta \alpha}\right\rfloor \delta \alpha, q\right) \\
& +\left(\frac{\eta^{+}}{\delta \alpha}-\left\lfloor\frac{\eta^{+}}{\delta \alpha}\right\rfloor\right)\left(\varphi\left(t, \alpha+\left\lceil\frac{\eta^{+}}{\delta \alpha}\right\rfloor \delta \alpha, q\right)-\varphi\left(t, \alpha+\left\lfloor\frac{\eta^{+}}{\delta \alpha}\right\rfloor \delta \alpha, q\right)\right)
\end{aligned}
$$

and

$$
\begin{aligned}
\mathcal{I}^{-} \varphi(t, \alpha, q)= & \varphi\left(t, \alpha-\left\lfloor\frac{\eta^{-}}{\delta \alpha}\right\rfloor \delta \alpha, q\right) \\
& -\left(\frac{\eta^{-}}{\delta \alpha}-\left\lfloor\frac{\eta^{-}}{\delta \alpha}\right\rfloor\right)\left(\varphi\left(t, \alpha-\left\lceil\frac{\eta^{-}}{\delta \alpha}\right\rfloor \delta \alpha, q\right)-\varphi\left(t, \alpha-\left\lfloor\frac{\eta^{-}}{\delta \alpha}\right\rfloor \delta \alpha, q\right)\right) .
\end{aligned}
$$

We define the numerical solution $h^{\delta t, \delta \alpha}: \mathbb{T}_{\delta t} \times \mathbb{R}_{\delta \alpha} \times([-\bar{Q}, \bar{Q}] \cap \mathbb{Z}) \rightarrow \mathbb{R}$ as follows,

$$
\begin{cases}h^{\delta t, \delta \alpha}(T, \alpha, q) & =q\left(-\operatorname{sign}(q) \Upsilon_{M O}-\psi q\right) \\ h^{\delta t, \delta \alpha}(n \delta t, \alpha, q) & =\mathcal{S}^{\delta t, \delta \alpha}\left(t, \alpha, q, h^{\delta t, \delta \alpha}((n+1) \delta t, \alpha, q)\right)\end{cases}
$$

Next we prove the convergence of 41). We first prove monotonicity, stability, and consistency properties (Propositions 1, 2 and 3 respectively) of $\mathcal{S}^{\delta t, \delta \alpha}(t, \alpha, q, \varphi)$. Combined with the comparison principle (Theorem 2), we prove the convergence; see Barles and Souganidis (1991). 
Lemma 1. The value function $H$ admits the following bounds:

$$
x+q S-|q| \Upsilon_{M O}-\psi q^{2} \leq H \leq x+q S+\left(\lambda^{+}+\lambda^{-}\right)(T-t) \Upsilon_{L O}+(T-t)(A+\theta) \sigma \bar{Q} .
$$

Proof.

$$
\begin{aligned}
& H(t, x, S, \alpha, q) \\
= & \sup _{\nu \in \mathcal{A}} \mathbb{E}_{t, x, S, \alpha, q}\left[X_{T}^{\nu}+Q_{T}^{\nu}\left(S_{T}^{\nu}-\operatorname{sign}\left(Q_{T}^{\nu}\right) \Upsilon_{M O}-\psi Q_{T}^{\nu}\right)-\phi \int_{t}^{T}\left(Q_{s}^{\nu}\right)^{2} \mathrm{~d} s\right] \\
= & x+q S \\
+\sup _{\nu \in \mathcal{A}} \mathbb{E}_{t, x, S, \alpha, q}[ & \int_{t}^{T} \mathrm{~d} X_{u}^{\nu}+\int_{t}^{T} S_{u}^{\nu} \mathrm{d} Q_{u}^{\nu}+\int_{t}^{T} Q_{u}^{\nu} \mathrm{d} S_{u}^{\nu}-\left|Q_{T}^{\nu}\right| \Upsilon_{M O}-\psi\left(Q_{T}^{\nu}\right)^{2} \\
& \left.-\phi \int_{t}^{T}\left(Q_{s}^{\nu}\right)^{2} \mathrm{~d} s\right] \\
=x+q S & {\left[\int_{t}^{T}\left(S_{u}^{\nu}+\Upsilon_{L O}\right) \mathrm{d} N_{u}^{+, l}-\int_{t}^{T}\left(S_{u}^{\nu}-\Upsilon_{L O}\right) \mathrm{d} N_{u}^{-, l}\right.} \\
+\sup _{\nu \in \mathcal{A}} \mathbb{E}_{t, x, S, \alpha, q} & -\int_{t}^{T}\left(S_{u}^{\nu}+\Upsilon_{M O}\right) \mathrm{d} M_{u}^{+}+\int_{t}^{T}\left(S_{u}^{\nu}-\Upsilon_{M O}\right) \mathrm{d} M_{u}^{-} \\
& +\int_{t}^{T} S_{u}^{\nu} \mathrm{d} N_{u}^{-, l}-\int_{t}^{T} S_{u}^{\nu} \mathrm{d} N_{u}^{+, l}+\int_{t}^{T} S_{u}^{\nu} \mathrm{d} M_{u}^{+}-\int_{t}^{T} S_{u}^{\nu} \mathrm{d} M_{u}^{-} \\
& \left.+\int_{t}^{T} Q_{u}^{\nu} \mathrm{d} S_{u}^{\nu}-\left|Q_{T}^{\nu}\right| \Upsilon_{M O}-\psi\left(Q_{T}^{\nu}\right)^{2}-\phi \int_{t}^{T}\left(Q_{s}^{\nu}\right)^{2} \mathrm{~d} s\right] .
\end{aligned}
$$

After cancelling terms, we have

$$
\begin{aligned}
& H(t, x, S, \alpha, q) \\
& =x+q S \\
& +\sup _{\nu \in \mathcal{A}} \mathbb{E}_{t, x, S, \alpha, q}\left[\Upsilon_{L O}\left(N_{T}^{+, l}-N_{t}^{+, l}\right)+\Upsilon_{L O}\left(N_{T}^{-, l}-N_{t}^{-, l}\right)\right. \\
& -\Upsilon_{M O}\left(M_{T}^{+}-M_{t}^{+}\right)-\Upsilon_{M O}\left(M_{T}^{-}-M_{t}^{-}\right) \\
& \left.+\int_{t}^{T} Q_{u}^{\nu} \mathrm{d} S_{u}^{\nu}-\left|Q_{T}^{\nu}\right| \Upsilon_{M O}-\psi\left(Q_{T}^{\nu}\right)^{2}-\phi \int_{t}^{T}\left(Q_{s}^{\nu}\right)^{2} \mathrm{~d} s\right] .
\end{aligned}
$$

For the lower bound, restrict the set of admissible strategies, such that the market maker sends 
MOs to clear out their position. In this case, we have

$$
H(t, x, S, \alpha, q) \geq x+q S-|q| \Upsilon_{M O}-\psi q^{2}
$$

For the upper bound, drop all the non-positive terms and write

$$
\begin{aligned}
& H(t, x, S, \alpha, q) \\
& \leq x+q S+\sup _{\nu \in \mathcal{A}} \mathbb{E}_{t, x, S, \alpha, q}\left[\Upsilon_{L O}\left(N_{T}^{+, l}-N_{t}^{+, l}\right)+\Upsilon_{L O}\left(N_{T}^{-, l}-N_{t}^{-, l}\right)+\int_{t}^{T} Q_{u}^{\nu} \mathrm{d} S_{u}^{\nu}\right] \\
& \leq x+q S+\left(\lambda^{+}+\lambda^{-}\right)(T-t) \Upsilon_{L O}+(T-t)(A+\theta) \sigma \bar{Q} .
\end{aligned}
$$

Proposition 1. (Monotonicity) For any $\delta t<f(\delta \alpha)$ for some $f:[0, \infty) \rightarrow[0, \infty), \varphi_{1}, \varphi_{2} \in$ $C_{b}^{1,2}([0, T] \times \mathbb{R} \times([-\bar{Q}, \bar{Q}] \cap \mathbb{Z}))$ such that $\varphi_{1} \leq \varphi_{2}$, we have $\mathcal{S}^{\delta t, \delta \alpha}\left(t, \alpha, q, \varphi_{1}\right) \leq \mathcal{S}^{\delta t, \delta \alpha}\left(t, \alpha, q, \varphi_{2}\right)$.

Proof. From expression (37), if

$$
\delta t<\left(\frac{\kappa A}{\delta \alpha}+\frac{\xi^{2}}{2 \delta \alpha^{2}}+\lambda^{+}+\lambda^{-}\right)^{-1}
$$

then $\mathcal{T}^{\delta t, \delta \alpha}(t, \alpha, q, \varphi)$ is monotone non-decreasing in $\varphi$. The monotonicity of $\mathcal{M}^{\delta t, \delta \alpha}(t, \alpha, q, \varphi)$ is obvious.

Proposition 2. (Stability) For any $\delta t>0$, there exists a unique solution $h^{\delta t, \delta \alpha}(t, \alpha, q)$ to (41). Furthermore, we have the uniform bounds

$$
L(q) \leq h^{\delta t, \delta \alpha}(t, \alpha, q) \leq U(t)
$$

where

$$
U(t)=(T-t)\left[\left(\lambda^{+}+\lambda^{-}\right) \Upsilon_{L O}+(A+\theta) \sigma \bar{Q}\right] \quad \text { and } \quad L(q)=-|q| \Upsilon_{M O}-\psi q^{2}
$$

Proof. Existence and uniqueness follows immediately from the definition of the explicit scheme (41). We first prove the upper bound. We notice that $h^{\delta t, \delta \alpha}(T, \alpha, q)=q\left(-\operatorname{sign}(q) \Upsilon_{M O}-\psi q\right) \leq$ 
$0=U(T)$, and

$$
\begin{aligned}
\mathcal{T}^{\delta t, \delta \alpha}(t, \alpha, q, U) & =U(t)+\delta t\left\{\bar{\alpha} \sigma q-\phi q^{2}+\left(\lambda^{+}+\lambda^{-}\right) \Upsilon_{L O}\right\} \\
& \leq U(t)+\delta t\left\{(A+\theta) \sigma \bar{Q}+\left(\lambda^{+}+\lambda^{-}\right) \Upsilon_{L O}\right\} \\
& =U(t-\delta t),
\end{aligned}
$$

and

$$
\mathcal{M}^{\delta t, \delta \alpha}(t, \alpha, q, U)=U(t)-\Upsilon_{M O} \leq U(t-\delta t)
$$

Thus, we have

$$
\mathcal{S}^{\delta t, \delta \alpha}(t, \alpha, q, U) \leq U(t-\delta t)
$$

As $h^{\delta t, \delta \alpha}(t-\delta t, \alpha, q)=\mathcal{S}^{\delta t, \delta \alpha}\left(t, \alpha, q, h^{\delta t, \delta \alpha}(t, \alpha, q)\right)$, we can prove by induction that $h^{\delta t, \delta \alpha}(t, \alpha, q) \leq$ $U(t)$.

The lower bound is attained by the strategy that immediately clears out any position and then does nothing until time $T$.

Proposition 3. (Consistency) For all $(t, \alpha, q) \in[0, T] \times \mathbb{R} \times([-\bar{Q}, \bar{Q}] \cap \mathbb{Z})$ and $\varphi \in C_{b}^{1,2}([0, T] \times$ $\mathbb{R} \times([-\bar{Q}, \bar{Q}] \cap \mathbb{Z}))$, we have

$$
\begin{aligned}
& \lim _{\substack{(\delta t, \delta \alpha) \rightarrow(0,0) \\
\left(t^{\prime}, \alpha^{\prime}\right) \rightarrow(t, \alpha)}} \frac{1}{\delta t}\left[\mathcal{T}^{\delta t, \delta \alpha}\left(t^{\prime}+\delta t, \alpha^{\prime}, q, \varphi\right)-\varphi\left(t^{\prime}, \alpha^{\prime}, q\right)\right] \\
& =\partial_{t} \varphi+\bar{\alpha} \sigma q-\kappa \bar{\alpha} \partial_{\alpha} \varphi+\frac{1}{2} \xi^{2} \partial_{\alpha \alpha} \varphi-\phi q^{2} \\
& \quad+\lambda^{+} \sup _{l^{+} \in\{0,1\}}\left[l^{+}\left(\Upsilon_{L O}+\varphi\left(t, \alpha+\eta^{+}, q-1\right)-\varphi\right)+\left(1-l^{+}\right)\left(\varphi\left(t, \alpha+\eta^{+}, q\right)-\varphi\right)\right] \\
& +\lambda^{-} \sup _{l^{-} \in\{0,1\}}\left[l^{-}\left(\Upsilon_{L O}+\varphi\left(t, \alpha-\eta^{-}, q+1\right)-\varphi\right)+\left(1-l^{-}\right)\left(\varphi\left(t, \alpha-\eta^{-}, q\right)-\varphi\right)\right] ;
\end{aligned}
$$

and

$$
\lim _{\substack{(\delta t, \delta \alpha) \rightarrow(0,0) \\\left(t^{\prime}, \alpha^{\prime}\right) \rightarrow(t, \alpha)}} \mathcal{M}^{\delta t, \delta \alpha}\left(t^{\prime}+\delta t, \alpha^{\prime}, q, \varphi\right)=\max \left\{\varphi(t, \alpha, q-1)-\Upsilon_{M O}, \quad \varphi(t, \alpha, q+1)-\Upsilon_{M O}\right\} .
$$


Proof. The limits converge by directly applying $\varphi \in C_{b}^{1,2}([0, T] \times \mathbb{R} \times([-\bar{Q}, \bar{Q}] \cap \mathbb{Z}))$.

Theorem 3. (Convergence) $h^{\delta t, \delta \alpha}(t, \alpha, q)$ converges locally uniformly to the unique viscosity solution $h(t, \alpha, q)$ as $(\delta t, \delta \alpha) \rightarrow(0,0)$, provided $\delta t<f(\delta \alpha)$ for some $f:[0, \infty) \rightarrow[0, \infty)$.

Proof. Follows Theorem 2, Propositions 1, 2, 3, and Barles and Souganidis (1991).

Define

$$
h_{*}(t, \alpha, q)=\liminf _{\substack{(\delta t, \delta \alpha) \rightarrow(0,0) \\\left(t^{\prime}, \alpha^{\prime}\right) \rightarrow(t, \alpha)}} h^{\delta t}(t, \alpha, q) \quad \text { and } \quad h^{*}(t, \alpha, q)=\limsup _{\substack{(\delta t, \delta \alpha) \rightarrow(0,0) \\\left(t^{\prime}, \alpha^{\prime}\right) \rightarrow(t, \alpha)}} h^{\delta t}(t, \alpha, q)
$$

which are, respectively, lower and upper semi-continuous functions on $[0, T] \times \mathbb{R} \times([-\bar{Q}, \bar{Q}] \cap \mathbb{Z})$, and inherit the boundedness of $h^{\delta t, \delta \alpha}(t, \alpha, q)$ by stability from Proposition 2. By definition, we have $h_{*} \leq h^{*}$. We claim that $h_{*}$ and $h^{*}$ are, respectively, a viscosity supersolution and a viscosity subsolution of (34), then by Theorem 2 (Comparison principle) we have $h^{*} \leq h_{*}$ and hence the equality. By symmetry, it suffices to show the viscosity supersolution property of $h_{*}$.

Let $(\tilde{t}, \tilde{\alpha}, \tilde{q}) \in[0, T) \times \mathbb{R} \times([-\bar{Q}, \bar{Q}] \cap \mathbb{Z})$ and $\varphi \in C_{b}^{1,2}([0, T] \times \mathbb{R} \times([-\bar{Q}, \bar{Q}] \cap \mathbb{Z}))$ such that $(\tilde{t}, \tilde{\alpha}, \tilde{q})$ attains the strict global minimum of $h_{*}-\varphi$. Then there exits a sequence $\left\{\left(t_{k}^{\prime}, \alpha_{k}^{\prime}, q_{k}^{\prime}\right)\right\}_{k} \in$ $[0, T) \times \mathbb{R} \times([-\bar{Q}, \bar{Q}] \cap \mathbb{Z})$ and $\left\{\left(\delta t_{k}, \delta \alpha_{k}\right)\right\}_{k}$, such that

$$
\begin{aligned}
\left(t_{k}^{\prime}, \alpha_{k}^{\prime}, q_{k}^{\prime}\right) & \rightarrow(\tilde{t}, \tilde{\alpha}, \tilde{q}), \\
\left(\delta t_{k}, \delta \alpha_{k}\right) & \rightarrow(0,0), \\
h^{\delta t_{k}, \delta \alpha_{k}} & \rightarrow h_{*}(\tilde{t}, \tilde{\alpha}, \tilde{q}),
\end{aligned}
$$

and $\left(t_{k}^{\prime}, \alpha_{k}^{\prime}, q_{k}^{\prime}\right)$ is the global minimizer of $h^{\delta t_{k}, \delta \alpha_{k}}-\varphi$.

We restrict $\left(\delta t_{k}, \delta \alpha_{k}\right)$ to satisfy the condition in Proposition 1, so that we can apply the monotonicity of $\mathcal{S}^{\delta t, \delta \alpha}$.

Define $\varepsilon_{k}=\left(h^{\delta t_{k}, \delta \alpha_{k}}-\varphi\right)\left(t_{k}^{\prime}, \alpha_{k}^{\prime}, q_{k}^{\prime}\right)$. Then by the numerical scheme in (41) and the monotonicity from Proposition 1, we have

$$
\begin{aligned}
& \varepsilon_{k}+\varphi\left(t_{k}^{\prime}, \alpha_{k}^{\prime}, q_{k}^{\prime}\right) \\
= & h^{\delta t_{k}, \delta \alpha_{k}}\left(t_{k}^{\prime}, \alpha_{k}^{\prime}, q_{k}^{\prime}\right) \\
= & \mathcal{S}^{\delta t_{k}, \delta \alpha_{k}}\left(t_{k}^{\prime}+\delta t_{k}, \alpha_{k}^{\prime}, q_{k}^{\prime}, h^{\delta t_{k}, \delta \alpha_{k}}\right) \\
\geq & \mathcal{S}^{\delta t_{k}, \delta \alpha_{k}}\left(t_{k}^{\prime}+\delta t_{k}, \alpha_{k}^{\prime}, q_{k}^{\prime}, \varphi+\varepsilon_{k}\right) \\
= & \mathcal{S}^{\delta t_{k}, \delta \alpha_{k}}\left(t_{k}^{\prime}+\delta t_{k}, \alpha_{k}^{\prime}, q_{k}^{\prime}, \varphi\right)+\varepsilon_{k} \\
= & \max \left\{\mathcal{T}^{\delta t_{k}, \delta \alpha_{k}}\left(t_{k}^{\prime}+\delta t_{k}, \alpha_{k}^{\prime}, q_{k}^{\prime}, \varphi\right), \mathcal{M}^{\delta t_{k}, \delta \alpha_{k}}\left(t_{k}^{\prime}+\delta t_{k}, \alpha_{k}^{\prime}, q_{k}^{\prime}, \varphi\right)\right\}+\varepsilon_{k} .
\end{aligned}
$$


After rearranging,

$$
\begin{aligned}
\max \left\{\frac{1}{\delta t_{k}}\left[\mathcal{T}^{\delta t_{k}, \delta \alpha_{k}}\left(t_{k}^{\prime}+\delta t_{k}, \alpha_{k}^{\prime}, q_{k}^{\prime}, \varphi\right)-\varphi\left(t_{k}^{\prime}, \alpha_{k}^{\prime}, q_{k}^{\prime}\right)\right]\right. \\
\left.\quad\left[\mathcal{M}^{\delta t_{k}, \delta \alpha_{k}}\left(t_{k}^{\prime}+\delta t_{k}, \alpha_{k}^{\prime}, q_{k}^{\prime}, \varphi\right)-\varphi\left(t_{k}^{\prime}, \alpha_{k}^{\prime}, q_{k}^{\prime}\right)\right]\right\} \leq 0 .
\end{aligned}
$$

Apply the consistency in Proposition 3 and let $k \rightarrow \infty$, to obtain

$$
\begin{aligned}
\max \left\{\begin{array}{l}
\partial_{t} \varphi+\bar{\alpha} \sigma q-\kappa \bar{\alpha} \partial_{\alpha} \varphi+\frac{1}{2} \xi^{2} \partial_{\alpha \alpha} \varphi-\phi q^{2} \\
+\lambda^{+} \sup _{l^{+} \in\{0,1\}}\left[l^{+}\left(\Upsilon_{L O}+\varphi\left(t, \alpha+\eta^{+}, q-1\right)-\varphi\right)+\left(1-l^{+}\right)\left(\varphi\left(t, \alpha+\eta^{+}, q\right)-\varphi\right)\right] \\
+\lambda^{-} \sup _{l^{-} \in\{0,1\}}\left[l^{-}\left(\Upsilon_{L O}+\varphi\left(t, \alpha-\eta^{-}, q+1\right)-\varphi\right)+\left(1-l^{-}\right)\left(\varphi\left(t, \alpha-\eta^{-}, q\right)-\varphi\right)\right] \\
\quad-\Upsilon_{M O}+\varphi(t, \alpha, q-1)-\varphi ; \\
\left.-\Upsilon_{M O}+\varphi(t, \alpha, q+1)-\varphi\right\} \leq 0
\end{array}\right.
\end{aligned}
$$

which is the viscosity supersolution property, as desired. Therefore, $h^{*} \leq h_{*}$, and hence $h^{*}=h_{*}$.

\section{Conclusions}

In this paper we derived the optimal strategy for a market maker who employs information of the drift of the asset to place limit orders and to send market orders to maximise expected profits. We referred to the drift of the asset as the alpha signal of the midprice, which follows a mean-reverting jump-diffusion process, where the jumps are caused by the arrival of market orders in the exchange.

We formulated the market maker's problem as a stochastic and impulse control problem and solved the corresponding HJBQVI numerically and proved the convergence of the numerical scheme. We provided maximum likelihood estimates of the model parameters using Nasdaq LOB data. We showed that when the alpha signal is near zero, the market maker tends to post both sell and buy limit orders (LOs) because the arrival rate of upward jumps in the midprice is approximately equal to the arrival rate of downward jumps in the midprice. As the alpha signal increases or decreases, the market maker posts LOs on one side of the LOB to avoid being picked

off on the wrong side of the LOB. Also, when the alpha signal increases (decreases) beyond a 
critical level, the market maker executes buy (sell) MOs in anticipation of an increase (decrease) in the price of the asset.

One of the challenges in the application of the optimal strategy derived here is that it requires almost instantaneous reaction to the change in the alpha signal, which has a half life much less than a second. Thus, markets makers will require software and hardware technology, in addition to being co-located close to the exchanges, to implement alpha trading strategies as well as any other high-frequency strategy; see Cartea and Sánchez-Betancourt (2018).

Our results also showed that the P\&L of a market maker who does not employ the alpha signal in their strategy will be lower than that of a market maker who employs the signal when the running inventory penalty parameter is low. As further research, one can explore how adverse selection and market making are affected when some market makers employ alpha signals in their strategy. Clearly, those who employ the alpha strategy will increase their profits, but those who do not employ the alpha signal will be adversely selected (i.e., picked off) by the market orders sent by those who employ the alpha strategy.

The alpha signal developed in this paper is relevant to all types of trading strategies, and not only to those of market makers. For example, those who execute a large trade over a time interval, will also benefit from incorporating alpha signals in their strategy; see, e.g., Cartea and Jaimungal (2016). Therefore, as above, the use of effective high-frequency strategies increases the adverse selection costs of other participants.

Finally, in our model, the market maker maximizes expected wealth and penalizes inventory holdings, which is a proxy to inventory risk. It would be interesting to consider performance criteria that penalize volatility in the terminal P\&L of the market maker's strategy and include other relevant market information (i.e., stochastic volatility of the midprice of the traded asset, clustering of market orders, limit order book volume imbalance). For example, the market maker could maximize the expected utility of terminal wealth. The solution to the problem would be similar to the one presented above. Note that we use numerical methods to solve the market maker's value function. In the same vein, the market maker could employ machine learning techniques to solve various flavours of the problem. 


\section{References}

Avellaneda, M. and S. Stoikov (2008). High-frequency trading in a limit order book. Quantitative Finance $\underline{8}(3), 217-224$.

Barles, G. and P. E. Souganidis (1991). Convergence of approximation schemes for fully nonlinear second order equations. Asymptotic analysis $\underline{4}(3), 271-283$.

Cartea, Á., R. Donnelly, and S. Jaimungal (2017). Algorithmic trading with model uncertainty. $\underline{\text { SIAM Journal on Financial Mathematics }}$ 8(1), 635-671.

Cartea, Á., R. Donnelly, and S. Jaimungal (2018). Enhancing trading strategies with order book signals. Applied Mathematical Finance, 1-35.

Cartea, Á. and S. Jaimungal (2015). Risk metrics and fine tuning of high-frequency trading strategies. Mathematical Finance 25(3), 576-611.

Cartea, Á. and S. Jaimungal (2016). Incorporating order-flow into optimal execution. Mathematics and Financial Economics 10(3), 339-364.

Cartea, Á., S. Jaimungal, and J. Penalva (2015). Algorithmic and high-frequency trading. Cambridge University Press.

Cartea, Á., S. Jaimungal, and J. Ricci (2014). Buy low, sell high: A high frequency trading perspective. SIAM Journal on Financial Mathematics 5(1), 415-444.

Cartea, Á., S. Jaimungal, and J. Ricci (2018). Algorithmic trading, stochastic control, and mutually exciting processes. SIAM Review 60(3), 673-703.

Cartea, Á. and L. Sánchez-Betancourt (2018). The shadow price of latency: Improving intraday fill ratios in foreign exchange markets. SSRN.

Guéant, O. (2016). The Financial Mathematics of Market Liquidity: From optimal execution to market making, Volume 33. CRC Press.

Guéant, O., C.-A. Lehalle, and J. Fernandez-Tapia (2013). Dealing with the inventory risk: a solution to the market making problem. Mathematics and financial economics 7(4), 477-507.

Ho, T., H. R. Stoll, et al. (1981). Optimal dealer pricing under transactions and return uncertainty. Journal of Financial Economics 9 (1), 47-73.

Øksendal, B. and A. Sulem (2007). Applied Stochastic Control of Jump Diffusions. Springer Science \& Business Media.

Seydel, R. C. (2010). Impulse control for jump-diffusions: Viscosity solutions of quasi-variational inequalities and applications in bank risk management. Ph. D. thesis, University of Leipzig. 